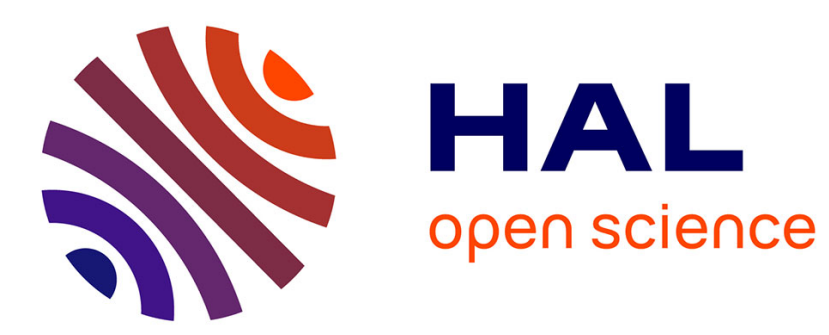

\title{
Premières formes de conditionnel chez l'enfant
}

Aliyah Morgenstern, Christophe Parisse

\section{To cite this version:}

Aliyah Morgenstern, Christophe Parisse. Premières formes de conditionnel chez l'enfant. Faits de langues, 2013, 40, pp.219-223. halshs-00793901

\section{HAL Id: halshs-00793901 https://shs.hal.science/halshs-00793901}

Submitted on 1 Mar 2013

HAL is a multi-disciplinary open access archive for the deposit and dissemination of scientific research documents, whether they are published or not. The documents may come from teaching and research institutions in France or abroad, or from public or private research centers.
L'archive ouverte pluridisciplinaire HAL, est destinée au dépôt et à la diffusion de documents scientifiques de niveau recherche, publiés ou non, émanant des établissements d'enseignement et de recherche français ou étrangers, des laboratoires publics ou privés. 


\title{
Premières formes de conditionnel chez l'enfant
}

\author{
Aliyah Morgenstern \& Christophe Parisse
}

\section{ACQUISITION DU SYSTEME TEMPOREL CHEZ L'ENFANT}

L'étude du développement des capacités linguistiques des enfants apporte un éclairage crucial sur les liens entre langage et cognition. Ces liens sont d'autant plus intéressants quand on travaille sur l'expression de la temporalité. Selon certains auteurs, le marquage des temps s'acquiert lentement et de manière graduelle chez l'enfant (Bronckart \& Sinclair 1973 ; Ferreiro 1991 ; Smith 1980) en raison de la combinaison de sa complexité syntaxique, sémantique et cognitive. Par ailleurs, l'enfant aurait moins l'occasion d'entendre parler d'évènements en rupture avec le moment d'énonciation que d'évènements présents et les formes temporelles référant au passé, au futur et au fictif seraient trop peu fréquentes dans le langage adressé pour que l'enfant puisse se les approprier durant les premières étapes de l'acquisition du langage (Tomasello 2003). Au niveau cognitif, l'enfant serait tout d'abord incapable de se décentrer et ne parlerait que de la situation et des actions en cours. Pour parler du passé, du futur ou d'évènements fictifs, l'enfant aurait besoin de pouvoir stabiliser ses représentations mentales et de les mettre en mots en l'absence d'ancrage direct dans la situation d'énonciation. Au niveau sémantique, les marqueurs temporels sont considérés d'après Reichenbach (1947) comme l'expression de la conscience des relations entre le moment de l'événement envisagé (point of event), le moment de la parole (point of speech) et le moment repère (point of reference). Selon Smith (1980), en raison de la complexité de ces relations, les enfants acquièrent très progressivement la capacité à marquer un décalage entre le moment de la parole et le moment de l'événement. Ce n'est que beaucoup plus tard ( 3 ans pour les enfants précoces, 5 ans pour les plus tardifs) qu'ils pourraient utiliser un repère temporel autre que le moment d'énonciation.

Morgenstern, Parisse et Sekali (2010) et Parisse et Morgenstern (2012) ont montré qu'effectivement, les premières formes utilisées en français par les enfants du Paris corpus sont le présent, le participe passé et l'infinitif qui sont également les premiers marqueurs d'aspect, et que dans un deuxième temps 
(entre 2;1 pour Madeleine, l'enfant la plus précoce et 2;11 pour Théophile, l'enfant le moins précoce), le système temporel va s'enrichir avec une variété de formes (y compris pour le même verbe): des marqueurs de passé (passé composé marquant l'antériorité, imparfait), de futur (futur périphrastique puis futur simple), d'irréel (subjonctif) ou de fictif (imparfait, conditionnel). Mais si les formes apparaissent dans un second temps, le codage dans le contexte dialogique des énoncés des enfants contenant des verbes a permis de montrer qu'ils sont peut-être capables d'exprimer des relations temporelles avant d'utiliser les marqueurs conventionnels de leur langue maternelle. Dès l'âge de 1;6, Anaé et Madeleine produisent quelques énoncés interprétés par leurs interlocuteurs et par les deux auteurs comme faisant référence au passé et au futur. Mais nous n'avons pas codé d'énoncés contenant des verbes interprétés comme faisant référence à du fictif avant l'emploi des temps habituellement employés pour cette fonction. Il reste donc tout à fait pertinent d'étudier les premiers emplois du conditionnel chez ces enfants et d'analyser les fonctions de ces formes en contexte dans nos corpus longitudinaux. Nous avons choisi pour cette courte étude de nous concentrer sur les deux enfants dont les données longitudinales recueillies et transcrites couvrent une période suffisamment longue pour étudier une large part du phénomène d'acquisition, Anaé et Madeleine.

\section{ANALYSE DES CONDiTIONNELS CHEZ MADELEINE ET ANAE DE 1;06 A 4;10}

De 1;06 à 2;01 pour Madeleine et à 2;02 pour Anaé, les deux petites filles utilisent beaucoup de présents et quelques passés composés et futurs périphrastiques ou des combinaisons de modaux suivis de l'infinitif. Dans un deuxième temps, les autres formes temporelles apparaissent, d'abord l'imparfait, puis le futur, puis le conditionnel (déjà à 2;01 pour la première fois dans notre corpus chez Madeleine et à 2;04 chez Anaé).

Le tableau 1 montre le nombre d'occurrences de conditionnels produits par Anaé et Madeleine en fonction de l'âge tout au long de notre corpus. Entre deux et trois ans, les deux petites filles produisent quelques occurrences (davantage pour Madeleine que pour Anaé). Entre 3 ans et 4 ans, elles peuvent toutes les deux produire une série de conditionnels dans des situations précises (jusqu'à 35 pour Madeleine à 4;01,27 alors qu'elle met en place un jeu fictif dans lequel elle attribue des rôles à chaque participant). Mais l'apparition relativement précoce des formes peut masquer l'existence de quatre étapes qui s'échelonnent entre $2 ; 01$ et $4 ; 10: 1$ ) une construction unique préférée assez fréquente ; 2) une diversification des formes; 3) des marquages d'hésitations et du travail de 
construction de la morphologie conventionnelle ; 4) la maîtrise de différentes fonctions du conditionnel.

\begin{tabular}{|c|c|c|c|c|c|c|c|}
\hline Madeleine & $\begin{array}{c}\mathrm{Nb} \text { occ. } \\
\text { cond. }\end{array}$ & $\begin{array}{c}\% \\
\text { cond. }\end{array}$ & $\begin{array}{c}\text { Nb total } \\
\text { de verbes }\end{array}$ & Anaé & $\begin{array}{c}\text { Nb occ. } \\
\text { cond. }\end{array}$ & $\begin{array}{c}\% \\
\text { cond. }\end{array}$ & $\begin{array}{c}\text { Nb total } \\
\text { de verbes }\end{array}$ \\
\hline $2 ; 01.02$ & 1 & $0,2 \%$ & 424 & $2 ; 01.05$ & 0 & $0,0 \%$ & 218 \\
\hline $2 ; 02.06$ & 2 & $0,4 \%$ & 530 & $2 ; 02.16$ & 0 & $0,0 \%$ & 357 \\
\hline $2 ; 03.05$ & 4 & $0,9 \%$ & 443 & $2 ; 03.30$ & 0 & $0,0 \%$ & 189 \\
\hline $2 ; 04.15$ & 2 & $0,3 \%$ & 583 & $2 ; 04.28$ & 1 & $0,3 \%$ & 288 \\
\hline $2 ; 05.12$ & 0 & $0,0 \%$ & 671 & $2 ; 05.30$ & 0 & $0,0 \%$ & 424 \\
\hline $2 ; 06.10$ & 2 & $0,6 \%$ & 349 & $2 ; 06.27$ & 0 & $0,0 \%$ & 416 \\
\hline $2 ; 07.07$ & 1 & $0,1 \%$ & 667 & $2 ; 08.04$ & 0 & $0,0 \%$ & 258 \\
\hline $2 ; 08.05$ & 3 & $0,5 \%$ & 643 & $2 ; 09.23$ & 0 & $0,0 \%$ & 271 \\
\hline $2 ; 09.16$ & 3 & $0,5 \%$ & 652 & $2 ; 10.24$ & 2 & $0,7 \%$ & 297 \\
\hline $2 ; 10.20$ & 1 & $0,3 \%$ & 396 & $2 ; 11.22$ & 0 & $0,0 \%$ & 318 \\
\hline $2 ; 11.19$ & 1 & $0,1 \%$ & 822 & $3 ; 01.07$ & 0 & $0,0 \%$ & 320 \\
\hline $3 ; 00.28$ & 4 & $0,7 \%$ & 602 & $3 ; 03.00$ & 1 & $0,3 \%$ & 335 \\
\hline \multirow[t]{2}{*}{$3 ; 03.02$} & 1 & $0,2 \%$ & 610 & $3 ; 04.27$ & 8 & $1,5 \%$ & 525 \\
\hline & & & & $3 ; 05.22$ & 1 & $0,3 \%$ & 371 \\
\hline $3 ; 06.08$ & 5 & $0,9 \%$ & 572 & $3 ; 06.27$ & 11 & $2,0 \%$ & 543 \\
\hline $3 ; 09.07$ & 2 & $0,4 \%$ & 488 & $3 ; 08.10$ & 0 & $0,0 \%$ & 323 \\
\hline \multirow[t]{2}{*}{$3 ; 09.28$} & 9 & $2,1 \%$ & 426 & $3 ; 10.00$ & 9 & $1,2 \%$ & 730 \\
\hline & & & & $4 ; 00.13$ & 8 & $1,1 \%$ & 707 \\
\hline $4 ; 01.27$ & 35 & $3,4 \%$ & 1029 & $4 ; 01.18$ & 0 & $0,0 \%$ & 511 \\
\hline $4 ; 07.04$ & 5 & $0,7 \%$ & 765 & $4 ; 04.10$ & 3 & $0,8 \%$ & 380 \\
\hline $4 ; 10.03$ & 8 & $2,0 \%$ & 404 & $4 ; 05.24$ & 2 & $0,6 \%$ & 344 \\
\hline
\end{tabular}

Tableau 1 - Production du conditionnel par Anaé et Madeleine.

(« Nb d'occ de cond. » correspond à « Nombre d'occurrences de conditionnels ». Le nombre total de verbes correspond au nombre d'occurrences de verbes par séance dans le corpus. Les adultes dans le corpus de Madeleine produisent en moyenne $1,04 \%$ de conditionnels par rapport au nombre de mots et $0,58 \%$ chez Anaé, ce qui est très proche de la moyenne des pourcentages que l'on trouve respectivement chez Madeleine $(1,3 \%)$ et Anaé $(0,54 \%)$ entre trois et quatre ans.)

\subsection{Des constructions préférées}

La première forme de conditionnel utilisée par Madeleine est « je voudrais » avec la valeur d' "atténuation» décrite par Dendale et Tasmowski (2001). Comme on a pu le montrer dans différentes études sur le développement linguistique de Madeleine (Leroy, Morgenstern \& Caët 2010 ; Morgenstern \& Sekali 2009), la mise en place des règles de politesse est importante dans cette 
famille et cette forme est très présente dans le langage entendu par l'enfant dans les échanges entre adultes ou dans le langage qui lui est adressé. Par exemple, quand Madeleine et sa mère jouent à la dinette (Madeleine 1;02), la mère lui dit «je voudrais un peu de gâteau » ou «je voudrais de l'eau». Elle lui pose également des questions en «tu voudrais » (« qu'est-ce que tu voudrais ?», « estce que tu voudrais du gâteau ?»). Par ailleurs, la construction est enseignée de manière délibérée à l'enfant :

Exemple 1 - Madeleine 2;03,5

*MAD: je veux écouter.

*PER: on dit pas je veux

*MAD: je voudrais! je voudrais écouter.

Il n'est donc pas très étonnant de trouver cette forme de mise à distance dans la requête : il s'agit de la forme utilisée et mise en place par l'entourage familial de l'enfant.

Chez Anaé le premier conditionnel utilisé à plusieurs reprises est « on dirait » avec la valeur d'éventualité de la catégorisation de Dendale et Tasmowski (op. cit). On trouve beaucoup d'occurrences (58) de cette construction dans le langage de la mère et des frères d'Anaé tout au long du corpus : « on dirait un robot non ? »; «on dirait qu'elle fait une galipette» (Anaé 2;0). Chaque séance en contient quelques-unes. Cette forme a une valeur sémantico-pragmatique très précise qui est parfois utilisée avec une mise en saillance en fin d'énoncé («c'est encore tes frères qui ont rangé ta bibliothèque, on dirait. »). Il s'agit de la seule forme de conditionnel utilisée par Anaé entre 2;04 et 3;04. Cette forme lui permet de chercher à identifier un objet et de donner une représentation alternative comme dans l'exemple suivant :

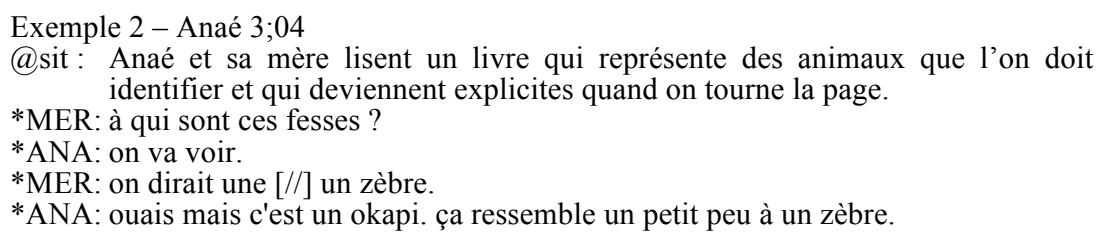

La mère d'Anaé dans cet extrait reconnaît le travail de recherche identificatoire d'Anaé («ouais ») puis souligne l'écart entre la mise en mots de la représentation d'Anaé et le nom du référent tel qu'elle peut l'identifier elle (« mais c'est un okapi ») tout en accordant une valeur de vérité à l'énoncé de sa fille («ça ressemble un petit peu à un zèbre »). La construction «on dirait» permet de poser une éventualité qui n'est pas remise en cause, il n'y a pas de conflit, il ne s'agit pas d'une valeur épistémique, d'une hypothèse puisque 
l'énoncé n'est pas réellement considéré comme réfutable. Il s'agit d'avancer un possible, de faire une suggestion en toute consensualité (marquée par le « on ») et la question n'est pas de décider si l'énoncé contenant «on dirait» est vrai ou faux, mais d'ajuster la représentation proposée au référent.

Il est très intéressant de voir que les premières formes de conditionnel produites par les deux enfants sont donc des constructions reprises à l'identique du langage de l'adulte et utilisées assez fréquemment avec une fonction extrêmement figée et dans les deux cas une valeur pragmatique identique à celle de l'adulte : la mise à distance d'une requête avec un effet d'atténuation et donc de politesse pour « je voudrais », la mise en place d'une suggestion consensuelle pour désigner des référents pour « on dirait ».

\subsection{Diversification}

Durant la période suivante qui arrive très vite pour Madeleine (2;04), et 12 mois plus tard pour Anaé (3;04), les formes vont se diversifier avec un certain nombre de verbes modaux en particulier. Madeleine produit des énoncés tels que « je devrais m'asseoir» à 2;04, « comme ça je pourrais... » et Anaé produit des énoncés au conditionnel également plus variés «il faudrait l'appeler » à 3;05, « on pourrait le regarder cet après-midi » en parlant d'une vidéo qu'elle adore regarder à 3;06 ou « on pourrait mettre des ailes à moi » à 3;10 pour expliquer comment il lui serait possible de voler. Non seulement les lexèmes verbaux utilisés varient de plus en plus, mais le conditionnel prend véritablement pour fonction le fait de proposer une éventualité dans un plan fictif ou en décalage temporel. On trouve également des formes de discours rapporté :

Exemple 3 - Anaé 3;10

*ANA: $<$ bah je $>[/ /]$ bah [/] bah [/] bah $<$ tu $\mathrm{m}^{\prime}>[/]$ tu m'aides ! pa(r)ce+que tu as dit que tu m'aid(e)rais !

*MER: bah pourquoi [/] pourquoi i(l) faut qu(e) je t' aide ?

L'enfant repère son énoncé par rapport à un temps $T$ («tu as dit») qui est en rupture par rapport au moment d'énonciation. Il s'agit d'une certaine forme de futur dans le passé, mais qui est médiatisée par le fait de rapporter le discours de l'autre.

\subsection{Marquages d'hésitations et de travail énonciatif}

On trouve, après cette période de diversification qui va de 2;04 à 3;03 chez Madeleine, et de 3;04 à 4;0 chez Anaé, une petite période de tâtonnement autour de la production de la morphologie du conditionnel. Les premières formes ont 
été produites de manière assez automatique dans une parfaite reprise des formes entendues dans le discours adulte. La deuxième période a permis aux enfants de diversifier leurs usages du conditionnel. Cet enrichissement de connaissances et la pratique du conditionnel les amènent à produire des formes nouvelles. Les deux petites filles opèrent un véritable travail de construction en ajoutant au lexème verbal des briques de morphologie parfois déformées, transformées sous l'effet de leur travail énonciatif. Il est particulièrement intéressant de constater que Madeleine, alors qu'elle produisait assez fréquemment des « je voudrais » en début de corpus dès 2;01, tâtonne à 3;03 et produit des énoncés dans lesquels elle fait des auto-rectifications telles que «tu sais moi je voula, je voudra avoir un nouveau ça, je voudra avoir un nouveau et je voudrais ça. » Par ailleurs, avec un verbe moins fréquemment utilisé au conditionnel elle produit à 4;01 un énoncé qui paraît assez confus en raison non seulement de son contenu mais de l'utilisation du verbe «appeler » avec et sans le réflexif et des deux sens associés («s'appeler Marie» et «appeler Marie»). Cet énoncé assez complexe comprends une véritable création morphologique : «Si Marie s'appelait Marie et moi je m'appelais Marie, toi t'appelais Marie et du coup, moi je venrais et toi tu appellerais Marie, Marie ma grande sœur. » L'énoncé marque la confusion que fait Madeleine entre l'imparfait et le conditionnel puisqu'elle dit «toi t'appelais Marie» la première fois au lieu de «toi t'appellerait Marie » qu'elle utilise ensuite en reformulant ce qu'elle vient de dire. «Venrais » est sans doute construit à partir de l'imparfait avec une valeur sémantique très voisine puisqu'il s'agit de créer un plan fictif dans lequel elle est une petite fille qui porte le même nom que sa propre grande sœur (Marie). Il y a donc une sorte de mélange ou d'assimilation entre les formes de conditionnel et d'imparfait hypothétique. Cependant, de manière générale tout au long des séances, l'imparfait sert plutôt à construire un cadre fictif et à raconter des évènements qui se déroulent dans un monde inventé. On constate en effet que la petite fille utilise en général l'imparfait pour expliquer les rôles et les actions de chacune dans le jeu fictif («je te donnais un doudou, je te mettais une couverture» à 4;01) et le conditionnel, toujours dans le plan du jeu fictif mais pour construire des hypothèses : dans son tâtonnement pour trouver la forme du conditionnel du verbe venir («venrais ») la petite fille pose clairement une hypothèse : si elle s'appelait Marie (comme sa grande sœur), chaque fois que sa mère appellerait Marie, c'est elle (Madeleine renommée Marie) qui viendrait, (mais ça n'irait pas puisque sa mère voudrait parler à sa grande sœur, d'où par la suite la solution adoptée - à l'imparfait - de les appeler Marie 1 et Marie 2).

\subsection{Maîtrise des formes et des fonctions}


En fin de corpus, les deux petites filles ne produisent plus de formes non standards et sont capables à la fois d'utiliser la fonction de futur dans le passé, de construction d'un plan fictif (Madeleine $4 ; 01$ «si on mettait comme ça, on ferait un thé »), de poser des hypothèses pour des besoins argumentatifs (Anaé 4;04 « parce qui sinon, y aurait quelqu'un qui volerait» ou Madeleine 4;04 « si elle était brûlante la neige, ça ferait brûlant »). Madeleine produit même en fin de corpus du conditionnel passé : il s'agit d'un véritable contrefactuel pour revenir sur une mauvaise manipulation qu'elle a faite en jouant à relier de petits éléments avec une ficelle.

Exemple 4 - Madeleine 4;10

*MAD: non pa(r)ce+que regarde <lui il est> [///] ça c'est dans c(e) sens. attends non j'aurais dû relier comme ça ! pa(r)ce que regarde lui il a une patte là et ça là lui là et lui là.

Madeleine est capable en fin de corpus d'utiliser un repérage en rupture avec le moment de l'énonciation : elle maîtrise donc des relations temporelles beaucoup plus complexes.

\section{CONCLUSION}

Le conditionnel, en dépit des faibles fréquences d'usage, se développe chez Anaé et Madeleine de la même façon que les autres formes temporelles, mais de manière un peu plus tardive. Son acquisition débute presque un an après celle des premières formes verbales, mais à 4 ans, l'enfant produit autant de conditionnels que l'adulte avec lequel elle est en interaction. Ce qui différencie le conditionnel des formes plus fréquentes est son usage. Les deux enfants l'utilisent au début avec une seule forme pour un seul verbe. Après une période de diversification qui amène Anaé et Madeleine à maitriser une variété de formes standards, elles procèdent alors à des essais morphologiques, des tentatives plus créatrices avec de nouveaux lexèmes verbaux, moins souvent entendus et maniés. A la fin de notre corpus, le conditionnel est utilisé de manière diverse et productive, sans toutefois recouvrir tous les usages potentiels de la langue française adulte.

Bronckart J.-P., and Sinclair, H. (1973). Time, tense and aspect. Cognition, 2: 107-130.

Dendale P. et Tasmowski L. (éds), (2001). Le conditionnel en français, Université de Metz : Recherches linguistiques 25.

Ferreiro, E. (1971). Les relations temporelles dans le langage de l'enfant. Genève: Droz.

Leroy, M. Morgenstern, A., Caët, S. (2010). L'auto-rectification chez l'enfant: appropriation et internalisation. La rectification, ouvrage en hommage à Mary-Annick Morel. Ophrys, Paris. 
Morgenstern, A., Parisse, C., and Sekali, M. (2009). A la source du futur : premières formes verbales dans les productions spontanées de deux enfants français de 18 mois à 3 ans. Faits de Langues: Le futur, 33 :163-175.

Morgenstern, A., Sekali, M. (2009). What can child language tell us about prepositions ? A contrastive corpus-based study of cognitive and socialpragmatic factors. Studies in Language and Cognition, Cambridge Scholars Publishing. Editors: Jordan Zlatev, Marlene Johansson Falck, Carita Lundmark and Mats Andrén. P. 261-275.

Parisse ,C. et Morgenstern, A. (2012). The unfolding of the verbal temporal system in French children's speech between 18 and 36 months. Numéro spécial sur First language acquisition of French grammar (éditeur Martine Sekali). Journal of French Language Studies, Vol 22, Issue 1. Cambridge: Cambridge University Press.

Reichenbach, H. (1947). Elements of symbolic logic, Berkeley: University of California Press.

Smith, C. S. (1980). The acquisition of Time Talk: relation between child and adult grammar. Journal of Child Language, 7: 263-278.

Tomasello, M. (2003). Constructing a language, a usage-based theory of language acquisition, Cambridge, M.A.: Harvard University Press. 